\title{
Outpatient treatment of low-risk venous thromboembolism with monotherapy oral anticoagulation: patient quality of life outcomes and clinician acceptance
}

\author{
This article was published in the following Dove Press journal: \\ Patient Preference and Adherence \\ 15 April 2016 \\ Number of times this article has been viewed
}

\author{
Jeffrey A Kline ${ }^{1,2}$ \\ Zachary P Kahler ${ }^{1,3}$ \\ Daren M Beam ${ }^{1,2}$ \\ 'Department of Emergency \\ Medicine, ${ }^{2}$ Department of Cellular \\ and Integrative Physiology, Indiana \\ University School of Medicine, \\ Indianapolis, IN, ${ }^{3}$ Department of \\ Emergency Medicine, University \\ of South Carolina Greenville School \\ of Medicine, Greenville, SC, USA
}

Correspondence: Jeffrey A Kline Department of Cellular and Integrative Physiology, Indiana University School of Medicine, 720 Eskenazi Avenue, Indianapolis, IN 46202, USA

Tel + I 3 I7 8803869

Mob +| 317670 054|

Email jefkline@iu.edu
Background: Oral monotherapy anticoagulation has facilitated home treatment of venous thromboembolism (VTE) in outpatients.

Objectives: The aim of this study was to measure efficacy, safety, as well as patient and physician perceptions produced by a protocol that selected VTE patients as low-risk patients by the Hestia criteria, and initiated home anticoagulation with an oral factor Xa antagonist.

Methods: Patients were administered the Venous Insufficiency Epidemiological and Economic Study Quality of life/Symptoms questionnaire [VEINEs QoL/Sym] and the physical component summary [PCS] from the Rand 36-Item Short Form Health Survey [SF36]). The primary outcomes were VTE recurrence and hemorrhage at 30 days. Secondary outcomes compared psychometric test scores between patients with deep vein thrombosis (DVT) to those with pulmonary embolism (PE). Patient perceptions were abstracted from written comments and physician perceptions specific to PE outpatient treatment obtained from structured survey.

Results: From April 2013 to September 2015, 253 patients were treated, including 67 with PE. Within 30 days, 2/ 253 patients had recurrent DVT and 2/253 had major hemorrhage; all four had DVT at enrollment. The initial PCS scores did not differ between DVT and PE patients (37.2 \pm 13.9 and 38.0 \pm 12.1 , respectively) and both DVT and PE patients had similar improvement over the treatment period ( $42.2 \pm 12.9$ and $43.4 \pm 12.7$, respectively), consistent with prior literature. The most common adverse event was menorrhagia, present in $15 \%$ of women. Themes from patient-written responses reflected satisfaction with increased autonomy. Physicians' $(\mathrm{N}=116)$ before-to-after protocol comfort level with home treatment of PE increased $48 \%$ on visual analog scale.

Conclusion: Hestia-negative VTE patients treated with oral monotherapy at home had low rates of VTE recurrence and bleeding, as well as quality of life measurements similar to prior reports.

Keywords: anticoagulants, quality of life, patient preference, menorrhagia, patient outcome assessment, health care, comorbidity, hemorrhage, pulmonary embolism

\section{Introduction}

Widening use of monotherapy to treat venous thromboembolism (VTE), including deep vein thrombosis (DVT) and pulmonary embolism (PE), has facilitated the treatment of low-risk patients with VTE at home without the need for hospitalization. In our previous work, we reported the 1-year experience of a protocol (the "Indy protocol") 
that included 106 patients who were diagnosed with VTE (including 35 with PE) in the emergency department (ED). ${ }^{1}$ These patients were determined to be at low risk for adverse outcomes using the Hestia criteria and were discharged from the ED with treatment with rivaroxaban. We believe that clinicians continue to be cautious about discharging patients with PE, possibly because of concerns that a patient discharged with PE may return within a few weeks with cardiovascular collapse or major hemorrhage. ${ }^{2,3}$

In this manuscript, we report the efficacy (VTE recurrence) and safety (bleeding) outcomes, as well as results of psychometric testing from 2 years of experience with the Indy protocol with two comparisons: 1) outcomes of patients with PE to those who had isolated DVT; and 2) patients treated with the Indy protocol to published results of psychometric testing from prior literature for patients treated with heparin and a vitamin $\mathrm{K}$ antagonist (VKA). The rationale for the first comparison is that outpatient treatment of DVT is a widely recommended standard of care, ${ }^{4}$ but outpatient treatment of PE is a new concept. Given that PE can cause sudden death, some clinicians may be hesitant to adopt the practice of discharging patients with PE. Therefore, a head-to-head comparison of outcomes of patients with DVT and PE treated as outpatients may help inform clinicians on the risks and benefits of this outpatient treatment for PE. The rationale for the second comparison comes from the fact that home treatment with oral monotherapy is also a new concept, and we believe it is valuable to compare the quality-of-life survey results from the Indy protocol to published results of VTE patients treated with heparin-VKA. ${ }^{5,6}$ We also report the written thoughts and perceptions of patients about the protocol. Finally, we surveyed clinicians for their perceptions regarding their comfort, concerns, and knowledge about outpatient discharge of VTE patients.

Our quantitative hypotheses were as follows: that PE patients would not differ from patients with DVT in 1) their Charlson comorbidity index, 2) normalized physical component summary (PCS) score of the 36-Item Short Form Health Survey 36 (SF36), or 3) the 30-day frequency for the composite end point of either recurrent VTE or bleeding; and that psychometric testing would show quality of life measurements similar to previously reported data for VTE patients, assessed using the PCS for all VTE patients and the validated Venous Insufficiency Epidemiological and Economic Study Quality of life/Symptoms questionnaire (VEINES QoL/Sym) survey for DVT patients. ${ }^{5,6}$

\section{Methods Study design}

This was a preplanned outcomes study that was part of a published protocol for the immediate discharge and further management of low-risk VTE from the ED using a novel target-specific anticoagulant. ${ }^{1}$ The protocol was approved as a research study by the Indiana University Institutional Review Board and is a registered observational trial (NCT02079584). All patients gave written informed consent to participate. In the first year of the study, $100 \%$ of patients were referred solely from two academic EDs (Eskenazi Health and Methodist Hospital, both teaching hospitals with residencies in emergency medicine in Indianapolis, IN, USA). Both emergency departments employ board-certified emergency physicians and emergency medicine residents. In the second year of the protocol (starting June 2014), we began accepting outpatients from other clinics, primarily orthopedic surgery and trauma clinics.

\section{Study protocol}

All patients had image-confirmed DVT or PE. To refer a patient, clinicians had to either complete an electronic survey (described previously ${ }^{1}$ ) or contact a study author by telephone to confirm that the patient was at low risk using the Hestia criteria with two modifications: patients were excluded for $>2$ doses of intravenous narcotics, rather than the original requirement of "severe pain requiring intravenous narcotics $>24$ hours", and patients were excluded for a pulse oximetry reading $<95 \%$ instead of $<90 \%$. Patients with active malignancy were further risk stratified using the POMPE-C (prediction of mortality from pulmonary embolism with cancer) tool.?

Patients were prescribed rivaroxaban $15 \mathrm{mg}$ twice per day for 21 days, followed by a 1-month prescription for $20 \mathrm{mg}$ once per day. Access to medications was facilitated by our case management team, which consists of registered nurses. Patients returned to one of two clinics, held on the second and fourth Fridays of each month, with one visit scheduled at $\sim 3$ weeks of diagnosis and a follow-up visit scheduled at 3-6 months. The day-to-day operations of the clinics are run by pharmacists at one site and nurse practitioners at the other.

Patient-centered outcomes included data collected at three time points: 1) at diagnosis, including clot location, demographics, payer status, vital signs, past medical history, Charlson comorbidity index, and laboratory values; 2) at the initial clinic visit, including the Rand SF36 quality of life 
survey, the VEINEs QoL/Sym survey, and patient overall perception of health (on a scale of 1-10); 3) outcome data for the first 30 days after diagnosis, including any ED visits, hospital admission for any reason, VTE recurrence, demonstrated by image-proven thrombosis at a new location (limb or lung), and any major or clinically relevant nonmajor bleeding event, using previously described explicit definitions. $^{8}$

The psychometric testing listed here was obtained from as many patients as was reasonably possible within the constraints of personnel, time, and patient willingness. At the initial visit, a medical student participating in a research elective also administered a questionnaire to record patients' free text written responses to three questions: "Describe your overall reaction to being treated at home and the clot clinic", "What were your main concerns about being discharged home from the emergency department?" and "What would you like us to do differently?" The students administering the questionnaires were trained by the investigators on how to deliver the surveys, including instructions to tell patients that their responses were optional and had no bearing on their medical care. Students were instructed to give patients as much time as they needed to answer the questions in the privacy of their own room in clinic and to inform patients of the option of giving verbal answers.

Follow-up data were obtained as previously described, ${ }^{1}$ including data collected on a structured collection form from the patient in the clinic, supplemented by telephone calls by a research coordinator to the patient in the event of a missed clinic appointment, followed by medical record review. Patients were asked about repeat visits, VTE diagnosis, and bleeding. Medical records were also reviewed by an experienced research coordinator to extract data on returns to ED, readmissions, VTE recurrence, and bleeding incidence within 30 days after initial diagnosis. The coordinator used explicit definitions for each variable. A $10 \%$ random sample was assessed by a second observer, and interobserver variability was measured for the end points of VTE and bleeding; the observers were found to have $100 \%$ agreement.

To obtain perceptions from clinicians, we created a survey in REDCap that asked eight questions about their perceptions of the protocol. This was performed after 1 year of protocol implementation. Questions were designed to assess clinician perceptions of the protocol, including the impact on their comfort level and frequency of discharging patients with PE.

\section{Data analysis}

The PCS, Charlson, and VEINEs QoL data were recorded and the responses were converted into scores according to published methods. ${ }^{9-13} \mathrm{We}$ compared the means of the Charlson index, PCS, and VEINEs QoL between PE and DVT patients with an unpaired $t$-test. Changes in PCS and VEINEs QoL from immediately postdiagnosis to 3-6 month follow-up were compared with a paired $t$-test. Proportions of patients with revisits to the ED, admissions, VTE recurrence, and bleeding were compared between PE and DVT with 95\% confidence interval (CI) for the difference. This was primarily a qualitative study of all existing patients in the clinic, thus no formal sample size was calculated a priori.

Based upon prior literature, the study population was assumed to be similar to prior outpatient VTE populations for PCS and VEINEs QoL values within one standard error of the mean: for the PCS, $38 \pm 1$ at diagnosis and $42 \pm 1$ at

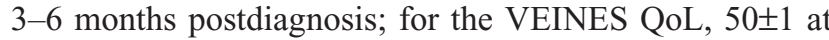
diagnosis and 55 $\pm 13-6$ months later. ${ }^{5,10}$ Survey results from clinicians are presented as descriptive data.

Patient free text responses were recorded in their own handwriting, and labeled with an identifier, and copied for coding. Coding was done by the three authors (ZPK, JAK, and DMB), who independently examined the written responses of patients and coded them using three criteria: relevance of the response to the clinic and VTE treatment (low or high), clarity of the point (low or high), and perceived importance (low or high), based upon frequency of expression by more than half of patients. Topics deemed not relevant included patient expressions about other medical providers unrelated to their problem, ED processes, or issues related to opiate pain medicines and neutral or nonresponsive comments (eg, "I just want to get better"). The three authors by consensus then selected excerpts that represented the credible themes, attempting to include both positive and negative perceptions.

\section{Results}

Figure 1 shows the flow diagram of patients enrolled. From April 2013 until September 2015, we enrolled 253 unique Hestia-negative VTE patients, including 67 with PE who were registered from either of the two participating EDs using the survey tool. Of the 253 patients, 209 (83\%) had at least one visit to our follow-up clinic, 35 (14\%) who followed up with a personal physician only, and nine (3.5\%) patients who were noncompliant with medical follow-up. We conducted paired (initial [2-4 week] and follow-up [3-6 months]) psychometric testing on 171 patients (67\%) and telephone and medical 


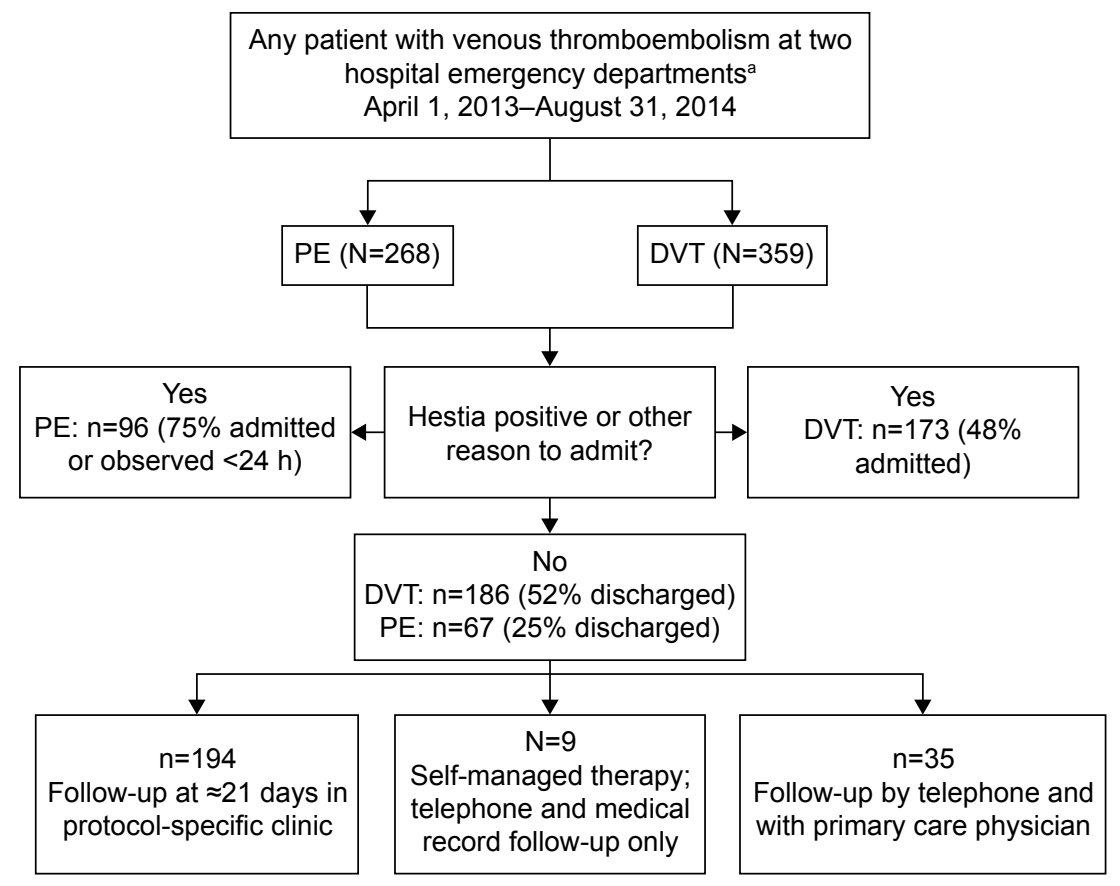

Figure I Flow diagram of patient enrollment and outcomes.

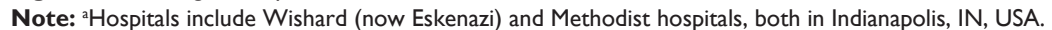

Abbreviations: DVT, deep vein thrombosis; h, hours; INPC, Indiana Network for Patient Care; PE, pulmonary embolism; SSDI, Social Security Death Index.

record follow-up for all 253 patients. Table 1 shows the vital signs and demographic data, including payer information. When mean values of demographics and vitals were compared for PE versus DVT (comparisons not shown), the only significant difference was that PE patients were younger than DVT patients $(41.5 \pm 14.8$ years versus $49.1 \pm 15.9$ years; $P=0.001$ from unpaired $t$-test). Patients were relatively young, with a high proportion of minorities and ethnicities, and about one-half had an income level at or below that required for

Table I Clinical and social features of the population

\begin{tabular}{lll}
\hline Continuous variables & Mean & Standard deviation \\
\hline Age, years & 47.0 & 16.1 \\
Heart rate, beats/min & 85.7 & 16.8 \\
Respiratory rate, breaths/min & 17.4 & 2.6 \\
Systolic blood pressure, $\mathrm{mmHg}$ & 137.0 & 24.1 \\
Diastolic blood pressure, $\mathrm{mmHg}$ & 82.3 & 15.0 \\
$\mathrm{SaO}_{2}, \%$ & 97.7 & 1.6 \\
$\mathrm{Body}_{\text {weight, } \mathrm{Kg}}$ & 100.8 & 43.9 \\
BMI, Kg/m & 35.5 & 15.3 \\
\hline Categorical variables & $\mathbf{N}$ & $\%$ of $\mathbf{2 5 3}$ \\
\hline Non-Caucasian race & 127 & $50 \%$ \\
Hispanic & 15 & $6 \%$ \\
Female & 113 & $45 \%$ \\
Medicaid & 101 & $40 \%$ \\
Self-pay & 36 & $14 \%$ \\
Medicare & 27 & $10 \%$ \\
PPO/private insurance & 87 & $34 \%$ \\
\hline Abbrevation
\end{tabular}

Abbreviations: BMI, body mass index; PPO, preferred provider organization.
Indiana Medicaid. Regarding the need for an ED-based protocol (as opposed to an office setting), the diagnosis of VTE was made between the hours of $7 \mathrm{pm}$ and 7 am in $62(25 \%)$ patients and on a weekend in $45(18 \%)$ patients.

Table 2 presents clot locations. Seventy-eight patients had clots in more than one anatomic site, including seven with both PE and DVT, and ten with bilateral leg DVT. Among the PE patients, 25 (37\% of PE) had bilateral PE, and 16 had isolated subsegmental PE (23\% of PE). Minor clot

Table 2 Clot characteristics

\begin{tabular}{lll}
\hline Location & $\mathbf{N}^{\mathbf{a}}$ & Percent of total \\
\hline Left leg & $9 \mathrm{I}$ & $36.0 \%$ \\
Right leg & 72 & $28.5 \%$ \\
Left arm & 5 & $2.0 \%$ \\
Right arm & 5 & $2.0 \%$ \\
Axillary vein & 1 & $0.4 \%$ \\
Calf vein & 45 & $17.8 \%$ \\
Femoral vein & $4 \mathrm{I}$ & $16.2 \%$ \\
Saphenous vein & 14 & $5.5 \%$ \\
Other vein & 30 & $11.9 \%$ \\
Left lung & 36 & $14.2 \%$ \\
Right lung & $5 \mathrm{I}$ & $20.2 \%$ \\
Subsegmental & 16 & $6.3 \%$ \\
Segmental or multiple subsegmental & 38 & $15.0 \%$ \\
Lobar or larger pulmonary artery & 13 & $5.1 \%$ \\
Unprovoked & 173 & $68.5 \%$ \\
\hline Note Sum
\end{tabular}

Note: asum of clot locations exceeds 253 because some patients had clots in more than one location. 
locations included isolated calf vein or isolated saphenous vein thrombosis, found in 23 patients (12\% of DVT). The majority $(69 \%)$ of clots were unprovoked.

Table 3 presents the frequency of abnormal laboratory values. The protocol required a basic metabolic package and complete blood count, but this was missing in $29 / 253$ (11\%) of patients. Forty percent of patients had elevated blood glucose, leukocytosis, or anemia. None had elevated troponin level, but three of 19 (16\%) had an elevated brain natriuretic peptide concentration.

Table 4 shows the comorbidities in the sample and demonstrates that the majority of patients $(67 \%)$ had a Charlson comorbidity index $\geq 1$. For the entire sample, the mean Charlson comorbidity index was $1.8 \pm 2.6$, and when compared between patients with PE and DVT, this value was not statistically significant $(1.4 \pm 2.4$ versus $2.0 \pm 2.0$; $P=0.146)$.

Table 5 presents data from the standard history and physical form used in the clinic, and only shows data for the first 30 days (ie, at the 2-4 week visit). The most common care process problem was difficulty in accessing the drug, in most cases related to need for preauthorization or the patient's inability to afford the copayment required for part D Medicare. The most frequent medical problem was menorrhagia, which occurred in 7\% of the entire population and $15 \%$ of women. Two patients among the $253(0.8 \%, 95 \%$ CI: $0.1 \%-1.9 \%$ ) had major hemorrhage, and two patients had recurrent VTE (both DVT) within 30 days.

\section{Psychometric testing}

Figures 2-4 compare the results of initial and follow-up psychometric testing for the 171 patients obtained in the clinic or by telephone follow-up between patients with $\mathrm{PE}(\mathrm{N}=65)$ and DVT $(\mathrm{N}=106)$. These 171 patients did not differ $(P>0.1$ unpaired $t$-test or chi-square test) for any variable in Table 1 or Table 4, compared with the 82 patients with missing data. Measurements were made at 3 weeks after diagnosis and then

Table 3 Abnormal laboratory values

\begin{tabular}{lll}
\hline Laboratory value & $\mathbf{n} / \mathbf{N}$ & $\%$ \\
\hline Troponin elevated $(>99$ percentile for CV <10\%) & $0 / 47$ & 0 \\
BNP $>90 \mathrm{pg} / \mathrm{mL}$ & $3 / 19$ & 16 \\
Blood glucose $>150 \mathrm{mg} / \mathrm{dL}$ in ED & $30 / 224$ & 13 \\
White blood count $>10,000 / \mu \mathrm{L}$ & $46 / 224$ & 21 \\
Hemoglobin $<10 \mathrm{~g} / \mathrm{dL}$ & $12 / 224$ & 5 \\
Creatinine $>1.5 \mathrm{mg} / \mathrm{dL}$ & $9 / 224$ & 4 \\
\hline
\end{tabular}

Abbreviations: BNP, brain natriuretic peptide; ED, emergency department; $\mathrm{n}$, number with laboratory value abnormal; $\mathrm{N}$, total number of laboratory done; $\mathrm{CV}$, coefficient of variability.
Table 4 Comorbid conditions and illness severity

\begin{tabular}{lll}
\hline Comorbidity component & $\mathbf{n}$ & \% of 253 \\
\hline Active malignancy & 7 & 3 \\
Smoker & 100 & 40 \\
Oral contraceptive use & 9 & 4 \\
Recent miscarriage & 1 & 0.4 \\
Postpartum status (<6 weeks) & 6 & 2 \\
COPD & 18 & 7 \\
Heart failure & 9 & 4 \\
Diabetes mellitus & 51 & 20 \\
Hypertension & 97 & 38 \\
CAD, no prior MI & 13 & 5 \\
CAD, prior MI & 6 & 2 \\
Chronic kidney disease, non-dialysis-dependent & 9 & 4 \\
Alcoholism & 14 & 6 \\
Charlson comorbidity index 0 & 83 & 33 \\
Charlson comorbidity index I-2 & 113 & 45 \\
Charlson comorbidity index 3-4 & 29 & 11 \\
Charlson comorbidity index $>4$ & 28 & 11 \\
\hline Abbreviations: CAD, coronary artery disease COPD & chronic & II
\end{tabular}

Abbreviations: CAD, coronary artery disease; COPD, chronic obstructive pulmonary disease; MI, myocardial infarction.

again at 3-6 months. Table 5 offers several salient points. First, in response to the question about overall health on a $1-10$ scale ( $1=$ worst, $10=$ best $),$ PE and DVT patients started at similar levels, but $\mathrm{PE}$ patients reported more improvement (from $3.5 \pm 2.5$ to $4.7 \pm 3.1$ ) than DVT patients (from $3.3 \pm 1.9$ to $3.2 \pm 2)$. Second, the first normalized VEINEs QoL result for our entire VTE population $(51 \pm 6)$ fell within the previously reported range of $50 \pm 1$ for warfarin-based regimens. ${ }^{5,10}$

Table 5 Patient-centered outcomes and complications within 30 days postdischarge

\begin{tabular}{lll}
\hline Outcome & $\mathbf{n}$ & \% of 253 \\
\hline Trouble getting drug? & 19 & 7.5 \\
Gum bleeding & 8 & 3.2 \\
Hematuria & 0 & 0.0 \\
Blood in stool & 3 & 1.2 \\
Easy bruising & 7 & 2.8 \\
Prolonged menstruation & 1 & 0.4 \\
Heavy menstruation & 17 & 6.7 \\
Unscheduled ED or clinic visit & 29 & 11.5 \\
Rehospitalization within 30 days & 4 & 1.6 \\
Repeat evaluation for DVT & 5 & 2.0 \\
Rehospitalization for recurrent DVT & $\mathrm{I}$ & 0.4 \\
Repeat evaluation for PE & 3 & 1.2 \\
Bleeding complications requiring hospitalization & 2 & 0.8 \\
Bleeding complications with transfusion of PRBCs & 2 & 0.8 \\
Rehospitalization for other diagnoses & 2 & 0.8 \\
Complications within 30 days (renal failure, liver & 0 & 0 \\
failure, stroke, and MI) & & \\
Death & 0 & 0 \\
\hline Note: Both patients satisfy the defition of maior bled. & & \\
\hline
\end{tabular}

Note: aBoth patients satisfy the definition of major bleed.

Abbreviations: DVT, deep vein thrombosis; ED, emergency department; MI, myocardial infarction; PE, pulmonary embolism; PRBC, packed red blood cells. 


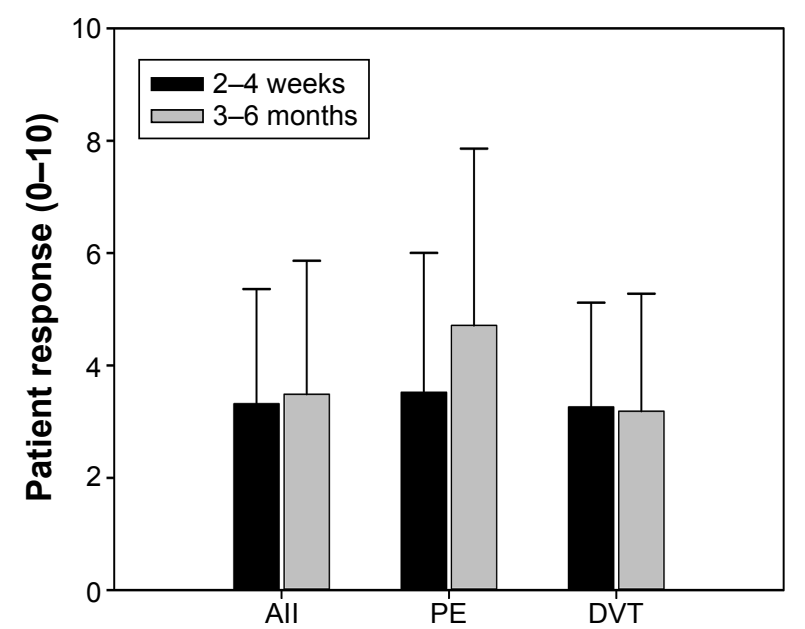

Figure 2 Patient response to question "On a scale of $\mathrm{I}-10$, with one being perfectly normal and ten being near death, how would you rate yourself?"

The first VEINEs QoL mean score was not different between PE vs DVT patients ( $P=0.13$ ), but those with DVT had larger increases on the second administration of the survey (from $48 \pm 6$ to $73 \pm 7 ; P<0.001$ paired $t$-test). Only seven patients with PE had diagnosed DVT. Third, the first PCS mean score for the entire population was $37.7 \pm 12.7$, which was within the predicted range of $38 \pm 1$ based on prior literature. The entire population showed a significant increase in PCS to $43.2 \pm 12.5$ on the second survey ( $P=0.021$, paired $t$-test $)$, at the upper end of the predicted value of $42 \pm 1$. Patients with PE and DVT showed similar increases in the PCS.

\section{Excerpts of patient statements}

The coders recognized three themes: 1) favorable reactions to the presence of a clot-focused clinic (65\% of perceptions); 2) positive perceptions about the anticoagulant medication, particularly surrounding the absence of required testing (55\%); and 3) concerns about why they had a clot, and questions about how to reduce the chances of future clots (48\%).

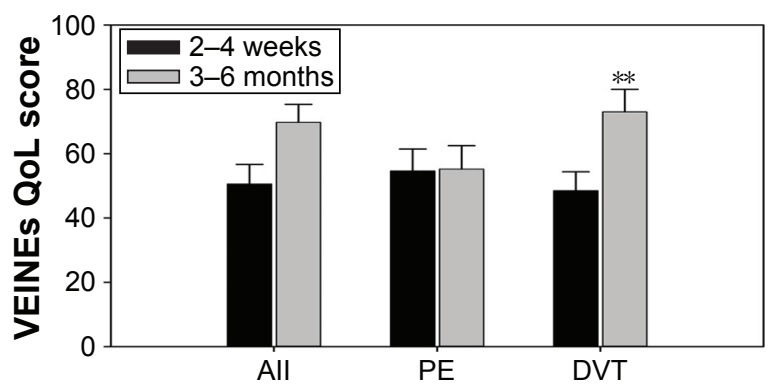

Figure 3 VEINEs QoL/Sym scores.

Note: ${ }^{* * P}<0.05$ vs $P E$, unpaired $t$-test; and $P<0.05$ vs $2-4$ weeks, paired $t$-test. Abbreviations: DVT, deep vein thrombosis; PE, pulmonary embolism; VEINEs QoL/Sym, Venous Insufficiency Epidemiological and Economic Study Quality of life/ Symptoms questionnaire.
Patients who had prior experience with warfarin also generally expressed positive statements about not having to take injections, with one notable dissenting patient expressing concern that she was not being "followed as closely". The authors selected nine quotes representative of these themes:

I was worried about not seeing the nurses in Coumadin clinic as often, but I felt better having the doctor's phone number, I can call anytime.

I don't stick my finger for my blood sugar, I'm sure not going to do it for my blood clot.

So I can go to New York for a month, and I don't have to follow up with anybody?

I believe Xarelto is better than taking coumadin blood thinners, being hospitalized, getting blood drawn on + off + whatever else comes with coumadin.

[...] I didn't want to stay in the hospital anymore. Thanks to this medicine I was able to go home. Glad that no needlesticks are required.

I believe that being treated at home was my best option.

I feel they saved me ... would much rather do this program instead of shots.

I am not worried about the next few months but am concerned about the long term.

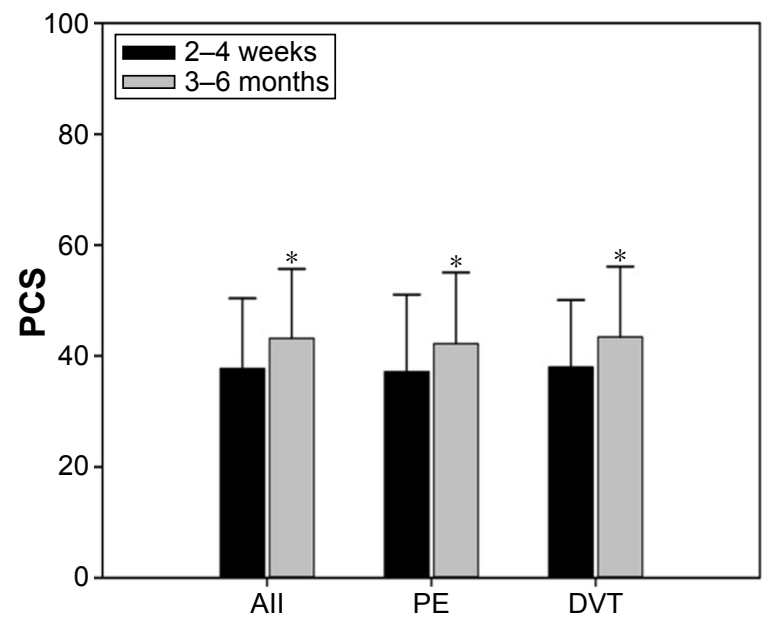

Figure 4 Normalized physical component summary (PCS) scores from the standard form 36 .

Note: $* P<0.05$ vs $2-4$ weeks, paired $t$-test.

Abbreviations: DVT, deep vein thrombosis; PCS, physical component summary; $\mathrm{PE}$, pulmonary embolism. 


\section{Physician survey}

We received responses from $89 \%$ of the clinicians surveyed $(\mathrm{n}=119)$. In response to the question "Prior to Clot Clinic (Indy Protocol), how comfortable would you have been discharging what you would consider a low-risk PE?", the mean ( \pm standard deviation) from the visual analog scale was $17.6 \pm 16.9 \mathrm{~mm}(0 \mathrm{~mm}=$ uncomfortable, $100 \mathrm{~mm}=$ highly comfortable); in response to the question "Would you currently feel comfortable sending home low-risk PE patients if they meet the Indy Protocol criteria?", this value increased to $66.0 \pm 25.6 \mathrm{~mm}$. Counts and frequencies of clinician responses to the question "If patients meet discharge criteria, what remaining concerns prevent you from sending them home? (Select all that apply)" were as follows: I haven't done it before/it's too unfamiliar: 48 (40.3\%); I'm not convinced the data support safe discharge: seven (5.9\%); I feel more comfortable if the patient is hospitalized: $25(21.0 \%)$; The patients I have approached don't want to go home: $14(11.8 \%)$; I need to discuss with an expert beforehand: nine (7.6\%); I'm too worried that I am medicolegally liable: 29 (24.4\%); Rivaroxaban might be too expensive: 39 (32.8\%); It is easier to admit than discharge these patients: $25(21.0 \%)$; I worry they need a thrombophilia workup: $12(10.1 \%)$; and I have no concerns if they meet criteria: 27 (22.7\%).

In response to the question "Of the ELIGIBLE patients you have seen in the past year, what percent have you APPROACHED about outpatient treatment?", the mean ( \pm standard deviation) of 119 responses was $82.1 \% \pm 24.8 \%$. Finally, the mean response to the question "Of the patients you have approached, what percent do you think have agreed to outpatient therapy?" was $81.5 \% \pm 25.0 \%$. Analysis of 63 free text responses revealed that $80 \%$ indicated the need for dedicated follow-up as the most important factor to their decision to treat $\mathrm{PE}$ at home.

\section{Discussion}

These data provide the first evidence of the safety, efficacy, and positive patient-centered outcomes associated with outpatient treatment of PE and DVT using monotherapy oral anticoagulation. Results indicate a low rate of VTE recurrence (two of the total 253, including zero of the 67 with PE) and major bleeding (also two of 253 total, including zero of 67 with PE) in the first 30 days after discharge. Psychometric scores were similar to those previously published in VTE patients treated with heparin and then oral VKA. ${ }^{5,6,10}$ Although the sample size is small, we provide preliminary evidence that psychometric outcomes were similar between patients with PE compared with patients with DVT.
When surveyed 1 year after implementation of the program, physicians expressed more comfort with discharging patients with PE, primarily because of assured follow-up. In the free text written comments, patients generally expressed appreciation and reduced anxiety because of the availability of a published protocol, a dedicated clinic, and ready access to the clinicians who run the clinic. Negative issues included problems with access to drug in $7.5 \%$ of patients, primarily related to problems with preauthorizations for private insurance and the "doughnut hole" in Medicare part D. We also emphasize that access to drug was facilitated by the use of case workers in the EDs who assisted in qualifying low-income, uninsured patients for a patient assistance program. Another potential concern was the high rate of menorrhagia after starting rivaroxaban, reported by $15 \%$ of women, requiring hysterectomy in one patient. In general, our approach to menorrhagia has been to recommend holding one dose of rivaroxaban, a strategy that we believe has been effective for most cases. No patient with menorrhagia required switching from rivaroxaban to another anticoagulant.

These data may help increase comfort level of patients and providers with outpatient treatment of PE. Our own survey data show that clinicians are hesitant to treat PE at home, despite most clinicians believing that home treatment of DVT is standard of care (level 1B recommendation from the American College of Chest Physicians). ${ }^{4}$ We believe that these data help justify the extension of this standard care for DVT to include home treatment of low-risk patients with PE. The data enable providers to inform patients with PE or DVT that if they are treated at home, their quality of life is likely to be as good as when they are hospitalized and is likely to improve with time. We also submit that the low rates of major hemorrhage and recurrent VTE comprise important information for patient education. Based upon prior work, patients are most concerned about the sequelae of hemorrhage (especially intracranial) and recurrent PE. ${ }^{14,15}$ Both our current patients' comments and the authors' collective experience argue for the pressing need for clinics that are dedicated to more than just the mechanical act of anticoagulation.

The dedicated clot clinic was important to the success of the Indy protocol. Clinicians indicated the need for follow-up as the most important component to their comfort with discharging low-risk patients. Implementation of the clinic was associated with a 50\% absolute increase in their expressed level of comfort. Likewise, more than one-half of patients wrote comments that indicated that they were specifically comfortable or satisfied with having a dedicated clinic for the care of their clots. The fact that other clinical specialties 
(orthopedic surgery and trauma surgery) have begun using the clinic points to successful dissemination.

Limitations include the lack of a randomized design, meaning that we do not have a matched comparator group. It could be hypothesized that if patients with PE (or DVT) had been hospitalized, it is possible that their initial psychometric testing would have been different. However, comparing our data to previously reported VTE psychometric testing reveals similar values for the PCS. ${ }^{5,10}$ This potential deficit is also being addressed in an ongoing randomized controlled trial (NCT02584660). The CIs for VTE recurrence and bleeding extend as high as 3\% in the PE subgroup. However, studies validating the Hestia criteria, ${ }^{16,17}$ including ours, ${ }^{1}$ have previously demonstrated safety. We only used rivaroxaban and have no data for other anticoagulants or outcomes in patients treated at home and cared for solely by other providers (eg, primary care provider).

\section{Conclusion}

Patients with acute DVT and PE diagnosed in the ED setting, who were deemed of low risk by objective criteria and were treated with monotherapy anticoagulation, had similar degrees of comorbidities, similar outcomes in terms of VTE recurrence and bleeding, and equal scores on psychometric tests that assessed quality of life. Physicians indicated marked increase in comfort level from before to after implementation of the protocol with discharging patients with acute PE. Patients generally expressed preference for home treatment with monotherapy anticoagulation for both DVT and PE. These data support the use of an outpatient treatment protocol for DVT and PE.

\section{Author contributions}

JAK conceived and designed the study, performed primary analysis, and wrote the first draft of the manuscript. DMB participated in development of the protocol and implementation of the protocol, wrote the manuscript, collected data, and performed analysis. ZPK edited the protocol, collected and analyzed the data, helped write, and edit the manuscript. All authors contributed toward data analysis, drafting and critically revising the paper and agree to be accountable for all aspects of the work.

\section{Disclosure}

In the past 3 years, JAK has received research grant funding from the Patient-Centered Outcomes Research Institute and the National Institutes of Health, Mallingkrodt in addition to being a consultant to Janssen Pharmaceuticals. The other authors report no conflicts of interest in this work.

\section{References}

1. Beam DM, Kahler ZP, Kline JA. Immediate discharge and home treatment of low risk venous thromboembolism diagnosed in two U.S. emergency departments with rivaroxaban: a one- year preplanned analysis. Acad Emerg Med. 2015;22:788-795.

2. Kabrhel C, Okechukwu I, Hariharan P, et al. Factors associated with clinical deterioration shortly after PE. Thorax. 2014;69:835-842.

3. Yoo HH, Queluz TH, El DR. Outpatient versus inpatient treatment for acute pulmonary embolism. Cochrane Database Syst Rev. 2014;11: CD010019.

4. Kearon C, Akl EA, Comerota AJ, et al; American College of Chest Physicians. Antithrombotic therapy for VTE disease: antithrombotic therapy and prevention of thrombosis, 9th ed: American College of Chest Physicians Evidence-Based Clinical Practice Guidelines. Chest. 2012; 141:e419S-e494S.

5. Kahn SR, Shbaklo H, Lamping DL, et al. Determinants of health-related quality of life during the 2 years following deep vein thrombosis. J Thromb Haemost. 2008;6:1105-1112.

6. Klok FA, van Kralingen KW, van Dijk AP, et al. Quality of life in long-term survivors of acute pulmonary embolism. Chest. 2010;138: 1432-1440.

7. Kline JA, Roy PM, Than MP, et al. Derivation and validation of a multivariate model to predict mortality from pulmonary embolism with cancer: the POMPE-C tool. Thromb Res. 2012;129:e194-e199.

8. Schulman S, Kearon CP; Subcommittee on Control of Anticoagulation of the Scientific and Standardization Committee of the International Society on Thrombosis and Haemostasis. Definition of major bleeding in clinical investigations of antihemostatic medicinal products in nonsurgical patients. J Thromb Haemost. 2005;3:692-694.

9. Hays RD, Sherbourne CD, Mazel RM. The RAND 36-item health survey 1.0. Health Econ. 1993;2:217-227.

10. Kline JA, Kabrhel C, Courtney DM, et al. Treatment of submassive pulmonary embolism with tenecteplase or placebo: cardiopulmonary outcomes at three months (TOPCOAT): multicenter double-blind, placebo-controlled randomized trial. J Thromb Haemost. 2014;12: 549-568.

11. Charlson ME, Pompei P, Ales KL, MacKenzie CR. A new method of classifying prognostic comorbidity in longitudinal studies: development and validation. J Chronic Dis. 1987;40:373-383.

12. Lamping DL, Schroter S, Kurz X, Kahn SR, Abenhaim L. Evaluation of outcomes in chronic venous disorders of the leg: development of a scientifically rigorous, patient-reported measure of symptoms and quality of life [see comment]. J Vasc Surg. 2003;37:410-419.

13. Kahn SR, Lamping DL, Ducruet T, et al; VETO Study investigators. VEINES-QOL/Sym questionnaire was a reliable and valid diseasespecific quality of life measure for deep venous thrombosis. $J$ Clin Epidemiol. 2006;59:1049-1056.

14. Locadia M, Bossuyt PM, Stalmeier PF, et al. Treatment of venous thromboembolism with vitamin K antagonists: patients' health state valuations and treatment preferences. Thromb Haemost. 2004;92:1336-1341.

15. HOGG K, Kimpton M, Carrier M, Coyle D, Forgie M, Wells P. Estimating quality of life in acute venous thrombosis. JAMA Intern Med. 2013;173: 1067-1072.

16. Zondag W, den Exter PL, Crobach MJ, et al; Hestia Study Investigators. Comparison of two methods for selection of out of hospital treatment in patients with acute pulmonary embolism. Thromb Haemost. 2013; 109:47-52.

17. Zondag W, Vingerhoets LM, Durian MF, et al; Hestia Study Investigators. Hestia criteria can safely select patients with pulmonary embolism for outpatient treatment irrespective of right ventricular function. J Thromb Haemost. 2013;11:686-692. 
Patient Preference and Adherence

Dovepress

\section{Publish your work in this journal}

Patient Preference and Adherence is an international, peer-reviewed, open access journal that focuses on the growing importance of patient preference and adherence throughout the therapeutic continuum. Patient satisfaction, acceptability, quality of life, compliance, persistence and their role in developing new therapeutic modalities and compounds to optimize

Submit your manuscript here: http://www.dovepress.com/patient-preference-and-adherence-journ clinical outcomes for existing disease states are major areas of interest for the journal. This journal has been accepted for indexing on PubMed Central The manuscript management system is completely online and includes a very quick and fair peer-review system, which is all easy to use. Visit http://www. dovepress.com/testimonials.php to read real quotes from published authors. 